\title{
Lernen im Dialog - Beschreibung und Analyse von Schülerdialogen beim Lösen eines Problems in einer Lerndyade
}

\section{Erwin Beck, Matthias Baer, Thomas Bachmann, Titus Guldimann, Ruth Niedermann und Michael Zutavern}

In der folgenden Studie wurden Dialoge untersucht, in denen zwei Schüler oder Schülerinnen ohne Hilfe einer steuernden und korrigierenden Lehrkraft gemeinsam versuchten, ein Problem zu lösen. Das Ziel, eine Alltagsfrage wie "Warum schwimmt ein Eisenschiff?" zu beantworten, weist den Weg zur Lösung des physikalischen Problems. Was im Dialog über das aufgerufene Sachproblem an Erfahrungen, Lösungsideen und Fragen zwischen den beiden Lernpartnern ausgelöst wird, macht die Fruchtbarkeit solcher Situationen aus. In einer repräsentativen Studie mit über 400 Schülerinnen und Schülern aus 20 Klassen des 8. Schuljahres wurden solche Dialoge $z u$ einer physikalischen und zu einer eher sprachlichen Problemstellung untersucht. Hier war das Ziel, Rahmenbedingungen zu analysierern, die beitragen, dass Dialoge mehr oder weniger lernförderlich verlaufen. Es konnten z.B. geschlechtsspezifische Unterschiede und die Bedeutung des Vorwissens nachgewiesen werden. Bei einer kleineren Stichprobe von 31 Dialogpaaren wurden die Äusserungen der Gespräche unter die Lupe genommen. Die Ergebnisse zeigten unter anderem, dass beim Lösen eines sprachlichen Problems eher die Fähigkeit zum Steuern eine Rolle spielt, bei der naturwissenschaftlichen Aufgabe die Vielfalt des sachlichen Vorwissens und "kreatives Abschweifen" helfen. Daraus lassen sich Folgerungen für das kooperative Lernen und die didaktische Bedeutung von Lernpartnerschaften ableiten. Ebenfalls thematisieren lässt sich die Bedeutung des Dialogs im gegenwärtigen Schulunterricht.

\section{Einleitung}

Das auf Vygotsky (1978) zurückgehende sozio-kulturelle Paradigma des Lernens löst immer neue Untersuchungen zur Bedeutung der Interaktion zwischen Lernenden aus. Entwicklungs- und lernpsychologisch interessant ist die Tatsache, dass nicht auschliesslich in der Interaktion zwischen einer Lehrperson und Lernenden gelernt werden kann, sondern durchaus auch unter Lernenden, die gemeinsam versuchen, eine Aufgabe oder ein Problem zu lösen (Doise, Mugny und Perret-Clermont, 1975; Perret-Clermont et al., 1976; Gilly, Fraisse und Roux, 1988; Brown und Palincsar et al., 1989; Slavin, 1990; Glaser, 1991; Beck et al., 
1992; Mercer, 1995; Brown und Campione, 1998; Fuchs et al., 1998; Lambert und McCombs, 1998; Baer, 1998).

Im Zuge der pädagogischen Forderungen nach selbsttätigem, schülerorientiertem und kooperativem Lernen erwachte das Interesse daran, die Bedingungen und Wirkungen dieses Lernens wissenschaftlich zu ergründen. In diesem Kontext lernpsychologischer und metakognitiver Erforschung des von Schülerinnen und Schülern selbstbestimmten und selbstregulierten Lernens entstand das Projekt «Lernen im Dialog». Bedeutsame Effekte, die in einer früheren Studie zum eigenständigen Lernen (Beck et al., 1992, 1995) in Lernpartnerschaften beobachtet wurden, sollten in lernenden und problemlösenden Dialogpaaren genauer untersucht werden.

Die Dialogforschung der siebziger Jahre (Wunderlich, 1969; Ehlich, 1981) war linguistisch geprägt. Sie analysierte schulischer Diskurse daraufhin, inwiefern gemeinsam diskutierendes Bemühen Erkenntnisse hervorbringt oder mindestens stark fördert. Neuere Studien konnten nachweisen, dass die Arbeit in Dyaden die Argumentationsfähigkeit der Partner und Partnerinnen begünstigt (Golder, 1992; Gilly und Deblieux, 1995). Mehr Aufschluss müsste man darüber haben, welche äusseren und inneren Faktoren in einem solchen Lerndialog zwischen Schülerinnen und Schülern Einfluss auf die gemeinsame Leistung und den individuellen Lernfortschritt ausüben. Beispielsweise sollten differenziertere Erkenntnisse über den Einfluss der Zusammensetzung von Dialogpaaren auf die gemeinsame Leistung gewonnen werden. Bekannt ist einiges über die Bedeutung einer guten Beziehung zwischen Dialogpaaren (Krappmann und Oswald, 1985). Ebenfalls bekannt waren die Versuche, die Wirksamkeit von Lerndialogen auf einer Makroebene zu bearbeiten. Dialogtypen - vom Quasidialog (Beck et al., 1997) bis zum explorativen (Dawes, Fisher und Mercer, 1993; Mercer, 1995) oder transaktiven Dialog (Berkowitz, 1982) - bezeichnen unterschiedliche Dialogqualitäten, indem sie das Scheitern oder Gelingen gemeinsamen Lernens im Dialog, das gegenseitige Verstehen oder eben auch das Aneinander-vorbei-Reden (Foppa, 1994) beschreiben.

In der dargestellten Studie geht es darum, erkenntnisfördernde und allenfalls auch hemmende Faktoren im dialogischen Lernen zu erfassen. Es geht dabei nicht um einen Vergleich zwischen Einzelarbeit und der gleichen Arbeit im Dialog, sondern um eine psychologische Analyse der Wirkfaktoren im Dialog. Es handelt sich nicht um eine Interventionsstudie. Die Leistungen der beiden Dialogpartnerinnen bzw. -partner im Problemlösedialog werden verglichen mit der im Vortest und im folgenden Nachtest geprüften Einzelleistung derselben Schülerinnen und Schüler. Es wurde nach äusseren und dialoginternen Bedingungen gesucht, die Unterschiede in den Leistungen erklären können.

Die Schülerinnen und Schüler löst in Dialogpaaren im Rahmen ihres normalen Unterrichts Problemaufgaben. Es fand kein Training statt. Die Lehrpersonen wurden nicht instruiert. Den Schülerinnen und Schülern wurde als eine von zwei Aufgaben die folgende Frage gestellt: «Warum schwimmt ein Eisenschiff?». 
Dies löste in den Dialogpaaren beispielsweise folgende Kommunikation aus ${ }^{1}$ :

A) Es kommt auf das Gewicht des Schiffs an.

B) Ja, aber ein Hochseeschiff, das ist ja Tonnen schwer.

A) Ich meine, es kommt auf das Gewicht an, ob es schwimmt.

B) Ja schon, aber das Gewicht allein ist es nicht, es ist auch die Form.

A) Was meinst du?

B) Man muss das Gewicht auf die Form so verteilen. (Der Schüler zieht beide Hände von der Mitte aus gegen aussen.)

A) Aber es geht noch nicht.

B) Aber wenn du einen Klumpen Gewicht hast, dann kannst du daraus eine immer grössere Form machen.

(...)

Die beiden Schüler versuchen im Dialog, eine für sie neue Erkenntnis zu schaffen. Dazu steht ihnen das je unterschiedliche Vorwissen und die Problemlösefähigkeit zur Verfügung. Im Dialog müssen die vorhandenen Kompetenzen sprachlich ausformuliert und die Denkprozesse sichtbar gemacht werden. Die oben erwähnte konvergente physikalische Aufgabe (Schiff-Problem mit einer physikalisch richtigen Lösung) wurde in der Untersuchung durch ein divergentes Überarbeitungsproblem (Song-Problem mit mehreren möglichen Lösungen) ergänzt. Die Schülerinnen und Schüler sollten die Übersetzung eines Popsongs verbessern. In beiden Situationen arbeiteten die Lernenden ohne Hilfe der Lehrperson. Die Lehrpersonen waren angewiesen worden, die beiden Themen nicht im Unterricht aufzugreifen.

Bei beiden Problemen waren folgende Fragen interesseleitend:

- Wodurch unterscheiden sich erfolgreiche von weniger erfolgreichen Dialogen?

- Welche äusseren Wirkfaktoren wie Alter, Geschlecht, Partnerzusammensetzung, usw. beeinflussen den Lernerfolg im Dialog?

- Welche inneren Wirkfaktoren wie Sachwissen, Interaktion, Problemlösen und Steuerung sind konstitutiv für erfolgreiche Dialoge?

\section{Theoretische Grundlagen und spezifische Fragestellung}

Die Untersuchungsanlage des Projekts «Lernen im Dialog» verbindet zwei entwicklungs- bzw. kognitionspsychologische Traditionen, jene des Genfers Jean Piaget und jene, die beim russischen Entwicklungstheoretiker Lew Vygotsky ihren Ursprung hat. Die gestellten Probleme, deren dialogische, teilweise von Handeln begleitete Lösung gefordert war, berücksichtigt einerseits die von Piaget (1936) hervorgehobene Bedeutung von kognitiven Konflikten für das Erkennen und die geistige Entwicklung des Individuums. Andererseits wird mit den Problemlöseund Lernprozessen, die sich in der dialogischen Auseinandersetzung entwickeln, 
Bezug genommen auf Vygotsky (1978), für den vor allem die sprachvermittelte soziale Interaktion für das Lernen und die Entwicklung des Einzelnen konstitutiv ist. Nach Vygotsky laufen die wichtigen Entwicklungsprozesse nicht bloss innerlich ab, sondern werden auf einer interpersonalen Ebene, die eine dialogische ist, vorbereitet.

Vollzieht sich die Problemlösung in der gegenseitigen Beachtung der vorgebrachten Argumente, so sprechen wir von einem «transaktiven Dialog» (Berkowitz, 1982). Ein transaktiver Dialog ist dadurch gekennzeichnet, dass beide Dialogpartnerinnen ein unvollständiges Vorwissen haben bzw. dass die vorliegende Aufgabe für beide ein Problem darstellt. Im Dialog verarbeiten sie wechselseitig eingebrachte Wissenselemente und Argumentationsweisen mit dem Ziel, gemeinsam eine Lösung zu finden. Dabei gehen sie substanziell auf gegenseitige Aussagen ein. Dies kann affirmativ geschehen, indem Wissen und Argumente, die jeweils von einer Partnerin eingebracht werden, von der anderen ergänzt, weitergeführt oder zu Ende gedacht werden. Der Dialog kann auch kontradiktorisch verlaufen, indem der eine Partner dem Wissen und den Argumenten des anderen widerspricht, nach Widerlegung sucht oder auf Widersprüchlichkeit und Unvollständigkeit hinweist.

Zusammenfassend können mit Glaser (1991) für Lernen im Dialog die folgenden Vorteile erwartet werden:

- Es steht mehr Wissen zur Verfügung, so dass verschiedene Problemlöseansätze und Schlussfolgerungen erwogen werden können.

- Gelegenheit für metakognitive, selbstregulative Aktivitäten werden vervielfältigt, da mehr kognitive Unsicherheiten und Dissonanzen auftreten.

- Zuhörende Partner beobachten und begleiten individuelle Denkprozesse, Meinungen und Vorstellungen (beliefs) und können Erklärungen beitragen, die Schwierigkeiten klären.

- Die Auseinandersetzung mit anderen Gesichtspunkten und Sichtweisen fordert zu einem gründlichen Verständnis der eigenen Position heraus.

Jeder leistet seinen Beitrag zur Problemlösung, was in der Begrifflichkeit Vygotskys dazu führen kann, dass eine "Zone der proximalen Entwicklung» entsteht, in der die gegenseitige Hilfe die Fähigkeiten der Dialogpartner zur Lösung des Problems steigert, was ohne Hilfe des Partners nicht möglich gewesen wäre. Die Dialogsituation stimuliert also Lern- und Entwicklungsprozesse und bewirkt kognitive Organisationsformen, die nicht vorkommen, wenn man alleine lernt (Perret-Clermont et al., 1976). Ebenso kann davon ausgegangen werden, dass die Interaktion unter Lernpartnern, die eine gute Beziehung zueinander haben, Dezentrierungen auslöst, was nach Piaget förderlich für die kognitive Entwicklung ist (z.B. Krappmann und Oswald, 1985).

Was aber passiert tatsächlich, wenn zwei Dialogpartner ein Problem bearbeiten? Was geht vor, wenn in einer Dyade - der kleinsten Einheit, von der sich der amerikanische Philosoph John Dewey fruchtbares Lernen versprach und worin er das Vorbild für die Zusammenarbeit in demokratischen Gesellschaften sah (De- 
wey, 1916) - gemeinsam ein Problem gelöst wird? Welche äusseren und inneren Wirkfaktoren spielen eine Rolle? Wie wirken sich Dialoge auf das fachliche und überfachliche Lernen aus - und was unterscheidet erfolgreiche von weniger erfolgreichen Dialogen?

Trotz reger pädagogisch-psychologischer Forschungstätigkeit zu Problemlösen und kooperativem Lernen sind diese Fragen noch wenig geklärt (Anderson et al., 1996).

\section{Ein heuristisches Modell von Lernen im Dialog und die Anlage unseres Forschungs- projektes}

Ausgangspunkt unserer Untersuchung ist das folgende heuristische Modell dialogischen Lernens:

Im Zentrum steht der Dialog zwischen zwei Schülerinnen oder Schülern. Formale und inhaltliche Dialogelemente tragen zur Konstituierung des Lernergebnisses bei, und der Dialog findet unter bestimmten personalen und situativen Rahmenbedingungen statt.

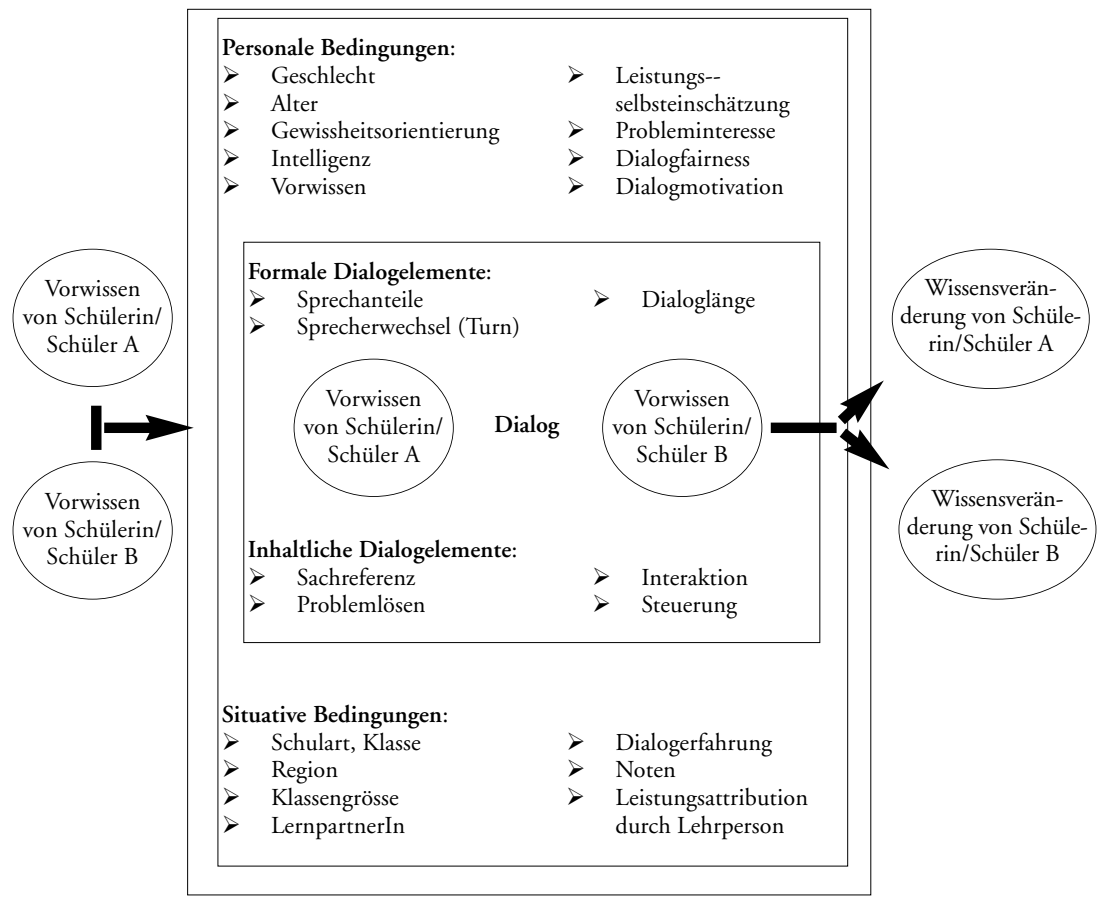

Abbildung 1: $\quad$ Heuristisches Modell dialogischen Lernens 
In einer Vorstudie mit vier Lehrpersonen wurden verschiedene Schlüsselprobleme zum Lernen im Dialog im Unterricht identifiziert, in Bezug auf die Rahmenbedingungen und die Aufgabenstellung analysiert und anschliessend erprobt (Beck, E. et al. 1998). Für die eigentliche Untersuchung wurden das Schiff- und das Songproblem ausgewählt. Die beiden Schlüsselprobleme beziehen sich auf unterschiedliche Gegenstandsbereiche und verkörpern zudem verschiedene Problemtypen.

An unserer Untersuchung beteiligten sich 20 repräsentativ für den Kanton St. Gallen ausgewählte Klassen des achten Schuljahres, insgesamt 187 Dialogpaare. In einem Vortest formulierte jede Versuchsperson ihre eigene Erklärung, warum ein Eisenschiff schwimmt. Am Ende eines etwa einstündigen Lerndialogs tat sie dies gemeinsam mit der Dialogpartnerin. Während des Dialogs zum Schiff-Problem standen zur handelnden Anregung und Überprüfung von Lösungsideen verschiedene Materialien und ein Behälter mit Wasser zur Verfügung. Im Nachtest wurde wieder eine individuelle Erklärung abgegeben. Ein Dialog galt als erfolgreich, wenn von Vor-zu Nachtest eine Leistungsverbesserung feststellbar war. Vor- und Nachtest lagen im Schnitt zwei Wochen auseinander. Die Ergebnisse dieser Untersuchungen führten zu Erkenntnissen über Rahmenbedingungen erfolgreicher Dialoge.

Zusätzlich wurde in jeder der 20 Klassen der Dialog zweier Paare auf Video aufgezeichnet und anschliessend transkribiert. Jede Sprechsequenz des einen Partners bis zur Ablösung durch den anderen wurde mindestens einer Kategorie eines Kategorisierungssystems, das in Vorversuchen entwickelt wurde, zugeordnet ${ }^{2}$. Anhand dieser detailliert ausgewerteten Dialoge konnte der Wirkung inhaltlicher Dialogelemente nachgegangen werden.

Beim Songproblem mussten die Dialogpaare die deutsche Rohübersetzung ( = wortwörtliche Übersetzung) eines englischen Songs («Dry county» von Bon Jovi) gemeinsam mit dem Ziel überarbeiten, dass der Text (a) in seiner globalen Textbedeutung verständlicher bzw. leichter rezipierbar wird und (b) zu Melodie und Rhythmik des Originalsongs gesungen werden kann. Diese - doppelte - Zielsetzung erfordert seitens der Dialogpaare die Auswahl und Anwendung unterschiedlicher Mittel und Strategien der Textüberarbeitung. Das Teilziel (a) fokussiert bzw. provoziert in erster Linie Textüberarbeitungs-Aktivitäten auf textsemantischer Ebene, das Teilziel (b) solche auf textsyntaktischer Ebene. Beide Teilziele konfrontieren die Dialogpaare mit einer divergenten - will heissen: offenen- Problemstellung. Weder mit Blick auf die «Verständlichkeit» der Übersetzung noch mit Blick auf deren "melodisch-rhythmische» Struktur gibt es - im Gegensatz zum Schiff-Problem - eine, und nur eine «richtige» Lösung.

Als Grundeinheiten für die qualitative Inhaltsanalyse der Lerndialoge legten wir die folgenden vier Hauptkategorien fest:

- Sachreferenz

- Problemlösen

- Interaktion

- Steuerung 
Die Kategorie Sachreferenz bezeichnet das inhaltsspezifische Wissen, das von den Dialogpartnerinnen zur Lösung des Problems beigezogen wurde. Beim SchiffProblem stellten wir sechzehn verschiedene Sachreferenzen oder Theoriebezüge fest. Die Propositionen sind von unterschiedlicher Qualität. Sie reichen von der unbestimmten, einen scheinbar allgemein bekannten und unbestreitbaren Tatbestand ansprechenden Aussage: "Man weiss doch... , dass ein Schiff schwimmt» bis zum physikalischen Begriff der Dichte, mit dem das Schwimmen eines Gegenstandes mit seiner geringeren Dichte gegenüber der Dichte des Wassers erklärt wird. Beim Song-Problem sind es die drei Sachreferenzen Stiltreue, Sinntreue und Adressatenbezug.

Die Kategorie Problemlösen erfasst in den Äusserungen kognitive Prozesse wie «Hypothesen über das Schwimmen aufstellen» oder «Erklärungen abgeben», die von den Dialogpartnerinnen im Verlaufe der gemeinsamen Problemlösung formuliert wurden. Auch hier ist die inhaltliche Ausprägung für das Schiff- und das Song-Problem verschieden. Beim Songproblem werden z.B. «Evaluierendes Lesen» von Übersetzungsteilen oder der "Vergleich von Textvarianten» als Problemlöseäusserungen erfasst.

Die dritte von uns untersuchte Kategorie heisst Interaktion. Sie bezieht sich auf den Partnerbezug und umfasst alle Aussagen, durch die sich die Dialogpartnerinnen gegenseitig in die Versuche, das Problem zu lösen, einbeziehen. Beim Schiff- und beim Song-Problem werden z.B. Weiterführungen der Argumente der Dyadenpartnerin oder Versuche, eine Behauptung des Partners zu widerlegen, ausgewertet.

Die Kategorie Steuerung schliesslich bestimmt im Lerndialog, welcher kognitive Prozess zu welchem Zeitpunkt im Fokus der Aufmerksamkeit steht. Hier werden ebenfalls die gleichen Faktoren bei beiden Problemen analysiert, z.B. Äusserung zur Aufgabensituation und Problemrepräsentation oder auch zur eigenen Leistungsfähigkeit.

\section{Ausgewählte Ergebnisse aus der Auswertung der Lerndialoge}

\section{Wirkfaktoren im Dialog}

\section{Schiff-Problem}

In der Detailstudie über 40 Dialoge konnten in einer Inhaltsanalyse vier Kategorienbündel untersucht werden: Sachreferenz, Problemlösen, Interaktion und Steuerung. Von diesen korrelierten beim Schiff-Problem zu unserer grossen Überraschung nur zwei Faktoren signifikant mit dem Dialogerfolg, nämlich ein Sachfaktor und ein Stimmungsfaktor.

Beim Sachfaktor handelt es sich um die Anzahl der in den Dialogen diskutierten unterschiedlichen Sachtheorien, also die Breite des von den Dialogpartnerinnen gemeinsam realisierten Sachwissens. 
Es bestätigte sich somit, dass erfolgreiche Dialogpaare mehr unterschiedliche Sachreferenzen aktualisieren, d.h. offenbar über mehr Wissen verfügen und dieses in der Problemsituation anwenden können, als weniger erfolgreiche. Dies belegt für die erfolgreichen Dialogpaare Glasers (1991) Vermutung, dass zwei dialogisierende Köpfe über ein differenzierteres Wissen verfügten als je ein Kopf allein. Dies wirkte sich in Form von verschiedenen Problemlöseansätzen und Schlussfolgerungen positiv auf das gemeinsame Problemlösen aus.

Beim Stimmungsfaktor - und das war wirklich überraschend - handelt es sich vorwiegend um spassige Äusserungen, die auf Sympathie zwischen den Dialogpartnern und -partnerinnen schliessen lassen und auf eine stimmungsmässig positive und entspannte Arbeitssituation hinweisen.

Wie aber ist zu erklären, dass gerade diese beiden Faktoren mit Dialogerfolg korrelieren?

Hier erweist sich das Modell von Abele über den Zusammenhang von Stimmung und Leistung als hilfreich. Abele legte kürzlich ihr Modell vor, in Weiterführung einer gestaltpsychologischen Idee aus den fünfziger Jahren (Abele, 1995; Metzger, 1957; Helm, 1954, 1958).

Zwischen Stimmung und Leistung vermitteln nach diesem Modell im Wesentlichen zwei Arten von Faktoren. Abele nennt sie kognitive und motivationale Mediatoren. Die Äusserungen, welche eine gute Stimmung bei den Dialogpartnern anzeigen und welche positiv mit Dialogerfolg korrelieren (Stimmungsfaktor), signalisieren einen günstigen Person-Umwelt-Bezug. Dies wirkt sich förderlich auf den Umfang und die Qualität des Informationsabrufs bei der Bearbeitung eines Problems aus (Sachfaktor). In guter Stimmung werden nach Abele mehr und verschiedenartigere Informationen abgerufen und verarbeitet.

Vor allem aber besteht bei guter Stimmung eine erhöhte Erfolgszuversicht und ein erhöhtes Zutrauen in die eigene Leistungsfähigkeit. Das Flow-Erleben ist gesteigert und der Gedankenfluss erleichtert (Csikszentmihalyi, 1991). Bei guter Stimmung liegt auch eher hohe Anstrengungsbereitschaft vor. Die Motivation, insbesonders die problembezogene Absichtsbildung, ist intrinsisch auf das Problem ausgerichtet. Extrinsische Bearbeitungsmotive, etwa der Wunsch, einer tieferen Bearbeitung des Problems auszuweichen, sind nicht vorhanden oder nur von geringer Bedeutung. Mehr Gedächtnisinhalte als in durchschnittlicher Stimmung sind gegenwärtig. Die Informationsverarbeitungsprozesse verlaufen «intuitiver», «vereinfachender», «lockerer», «flexibler», «freier verknüpfend» und stärker «anmutend-probierhaft als in durchschnittlicher oder negativer Stimmung», und es werden auch komplexe, ungewöhnliche Verknüpfungen gesehen (Abele, 1995). Erlebensbezogene Kognitionen, die auf die Bewältigung einer affektiv unangenehmen Situation gerichtet sind - also auf das eigene Erleben und nicht das Problem -, kommen nicht oder nur in geringem Ausmass vor.

Dies alles lässt sich plausibel auf die erfolgreichen Dialogpaare beziehen. Allerdings ist die Frage offen, was Ursache und was Wirkung ist: Ist es die gute Stim- 
mung im Dialog, die das Wissen «lockert», oder ist es das elaboriertere Wissen der Partner, das zu einer guten Stimmung im Dialog führt?

Nicht bestätigen können wir aufgrund unserer Resultate die für die Dialogsituation angestellte Vermutung, es würden mehr metakognitive und selbstregulative Aktivitäten auftreten. Das hätte sich in Korrelationen in unserem Kategorienbündel der Steuerung auswirken müssen. Ebenfalls nicht bestätigen lassen sich die von Glaser (1991) und Berkowitz (1982) vermutete Bedeutung der gegenseitigen produktiven Verknüpfung von Argumenten der Dialogpartnerinnen in Form von Transakten, denn dies hätte sich durch Korrelationen zwischen den von uns ausgewerteten Interaktionsfaktoren zeigen müssen.

\section{Song-Problem}

Von den mit dem Manual zur qualitativen Inhaltsanalyse von Lerndialogen untersuchten vier Kategorienbündel korrelierten beim Song-Problem die expliziten Steuerungsäusserungen in stärkerem Ausmass mit dem Dialogergebnis, wenngleich das Signifkanzniveau (knapp) nicht erreicht wurde. Im Übrigen besteht nur zwischen den aufgabenfremden Äusserungen und dem Dialogergebnis eine signifikante Korrelation, allerdings eine negative: je schlechter das Dialogergebnis, desto mehr aufgabenfremde Äusserungen gibt es. Im Unterschied zum SchiffProblem gefährdeten die aufgabenfremden Äusserungen beim Song-Problem die Lösung des Problems.

Wie aber lässt sich dieser Sachverhalt erklären?

Das Schiff- und das Song-Problem stellten offenbar für die Schülerinnen und Schüler, die in den Dialogpaaren arbeiteten, deutlich unterschiedlich schwierige Herausforderungen dar. Während die Dialogpaare beim Schiff-Problem in einem Aufgabenbereich arbeiteten, der ihnen in seiner inhaltlichen Art und in der Art der konvergenten Problemstellung durch die Erfahrungen im Physikunterricht vertraut war und sie überdies zu diesem Schlüsselproblem über relativ reichhaltiges physikalisches Vorwissen verfügten, mussten sie für die Bearbeitung des Song-Problems im doppelten Sinne Neuland betreten. Die wenigsten Dialogpaare hatten zuvor ein Problem der Art des Song-Problems bearbeitet. Das divergente SongProblem war deshalb ein im hohen Mass unvertrautes Problem, das zudem auf ungewohnte Art und Weise bearbeitet werden sollte. Zwar hatten die in den Dialogpaaren arbeitenden Schülerinnen und Schüler im Deutschunterricht auch schon divergente Probleme bearbeitet, z.B. beim Verfassen eines Aufsatzes, doch noch kaum je ein divergentes Problem in der Form einer Textübersetzung zu einer Melodie. Die Dialogpaare verfügten deshalb über keine entsprechenden Kenntnisse, die sie für die gemeinsame Bearbeitung des Song-Problems hätten beiziehen können. Sich in dieser Situation sinnvoll zu verhalten bedeutete, mit der Dialogpartnerin darüber zu sprechen, wie gemeinsam vorzugehen sei. Dies erklärt die relativ hohe Zahl der expliziten Steuerungsäusserungen.

Damit liesse sich die negative Korrelation der sachfremden Äusserungen mit dem Dialogerfolg im Sinne Abeles als ein Ausdruck der Verunsicherung und 
Überforderung der Schülerinnen und Schüler angesichts des neuartigen divergenten Problems interpretieren.

\section{Wissen erwerben im Dialog}

Ein Hauptinteresse des Projektes «Lernen im Dialog» zielte auf die Untersuchung des selbständigen Erwerbs von Wissen im Dialog. In der Kognitionspsychologie wurde der Erwerb von Wissen lange Zeit fast ausschliesslich als Auf- und Ausbau kognitiver Strukturen eines allein lernenden Individuums betrachtet. Zwar wird in der Lehr-Lernforschung der Erwerb von Wissen durch Instruktion untersucht, doch steht dabei die Vermittlung von Kenntnissen durch eine Lehrperson bzw. die Aufnahme des Wissens durch einen Lernenden im Zentrum. In letzter Zeit ist diese Einseitigkeit durch die zunehmende Berücksichtigung der Bedeutung des sozio-kulturellen Kontextes für das Lernen abgebaut worden (Pauli, 1998; Stebler, 1999). Beim dialogischen Lernen im transaktiven Sinne geht es um die gemeinsame Konstruktion von neuem Wissen von zwei Lernwilligen aufgrund ihrer individuellen Ressourcen und einer gemeinsamen Problemstellung.

Die im Vortest, in der Dialogsituation und im Nachtest abgegebenen individuellen und gemeinsamen Erklärungen zum Schiff-Problem wurden auf ihre sachliche Richtigkeit hin beurteilt und nach der jeweiligen Nähe zur physikalisch richtigen Erklärung rangiert. Insgesamt liessen sich sechs Niveaus unterscheiden (Tabelle 1).

\section{Tabelle 1: Die sechs qualitativ unterschiedlichen Erklärungsniveaus zur Frage "Warum schwimmt ein Eisenschiff?»}

\begin{tabular}{|c|c|}
\hline Gruppe 1: & $\begin{array}{l}\text { Erklärung fehlt oder ist nicht zu verstehen / animistische Erklärungen / Erklärungen, die das } \\
\text { Schwimmen auf einen Motor oder sonstigen Antrieb zurückführen. }\end{array}$ \\
\hline Gruppe 2: & $\begin{array}{l}\text { Das Schwimmen wird allein durch das Material des Schiffes, durch magnetische Kraft oder } \\
\text { Oberflächenspannung erklärt. }\end{array}$ \\
\hline Gruppe 3: & Luft im Schiff wird als der ausschlaggebende Faktor gesehen. \\
\hline Gruppe 4: & $\begin{array}{l}\text { Mit Erklärungen durch Gewicht, Masse, Form, Hohlraum, Volumen werden einzelne Elemente, } \\
\text { die für die richtige Erklärung notwendig sind, genannt und physikalische Begriffe verwendet - } \\
\text { diese Elemente jedoch noch nicht miteinander verbunden. }\end{array}$ \\
\hline Gruppe 5: & $\begin{array}{l}\text { In dieser Gruppe werden die in Gruppe } 4 \text { genannten Grössen in Verbindung miteinander gebracht } \\
\text { oder das Schwimmen durch «Verdrängung» oder «Druck» erklärt, ohne dass man aus den Erläute- } \\
\text { rungen der Schülerinnen und Schüler eine physikalisch korrekte Erklärung herauslesen könnte. }\end{array}$ \\
\hline Gruppe 6: & $\begin{array}{l}\text { Alle physikalisch richtigen Erklärungen mit Hilfe von Kraft oder Gewichtsvergleich bzw. Dichte } \\
\text { sind in dieser Gruppe zusammengefasst. }\end{array}$ \\
\hline
\end{tabular}

Niveau 1 enthält die fachinhaltlich schwächsten, Niveau 6 die anspruchvollsten Erklärungen. Auf Niveau 1 beispielsweise fehlt eine Erklärung, sie ist unverständlich, oder das Schwimmen wird animistisch ${ }^{3}$ oder durch einen Motor oder einen sonstigen Antrieb erklärt. Dagegen wird auf Niveau 3 Luft im Schiff als ausschlaggebend angesehen. Auf Niveau 4 werden Elemente der richtigen Erklärung wie Gewicht, Masse, Form, Hohlraum oder Volumen, genannt, ohne diese Elemente bereits miteinander zu verbinden. 
Die häufigsten Erklärungen kamen auf Niveau 4 vor. Warum ein Eisenschiff schwimmt, wurde somit vielfach durch eindimensionale Erklärungen, also durch Bezugnahme auf einen Faktor, etwa das Gewicht oder das Volumen, beantwortet. Das notwendige In-Beziehung-Setzen beider Faktoren gelang relativ wenigen.

Wie beschrieben wurde zum Thema kein Unterricht durchgeführt. Zwischen Vor- und Nachtest lag also nur der unbegleitete Dialog der Schülerinnen und Schüler. Im gemeinsamen Ergebnis von etwa 40\% der Schülerinnen und Schüler finden sich Erklärungen auf dem Niveau ihrer Vortests. Rund 25\% der Schülerinnen und Schüler regredierten in der Dyade auf ein niedrigeres Niveau, 35\% sind an einem Ergebnis beteiligt, das ihr Vorwissen übersteigt.

Eine vertiefende Analyse der Daten zeigt, dass 20\% der Schülerinnen und Schüler im Nachtest alleine das Problem auf einem Niveau erklären, das weder bei ihnen noch bei ihrem Gesprächspartner im Vortest zu beobachten war. Es bestätigt sich somit, dass zwei Lernende im Dialog neues Sachwissen konstruieren und somit Wissen erwerben können, über das sie zuvor allein noch nicht verfügten. In der Detailanalyse der 20 Dialoge konnten in einigen Gesprächen das Auftauchen solcher neuer, höherwertiger Sachäusserungen rekonstruiert werden.

\section{Zusammensetzung der Dialogpaare}

Krappmann und Oswald (1995) haben in ihren Feldstudien über die Kooperation von Schulkindern gezeigt, dass Sympathie eine wichtige Voraussetzung für erfolgreiches gemeinsames Lernen darstellt. Wir liessen deshalb in unseren Studien die Schülerinnen und Schüler unserer Versuchsklassen sich selbst zu Dialogpaaren zusammenfinden.

Aus entwicklungspsychologischen Studien in der Tradition Piagets (Glachan und Light, 1982; Doise und Mugny, 1984; Bell, Grossen und Perret-Clermont, 1985; Doise, 1990; zur Diskussion: Pauli, 1994) ist bekannt, dass ein minimaler Leistungsunterschied zwischen den Dialogpartnern eine für das Lernergebnis förderliche Bedingung ist. Keine Unterschiede oder zu grosse scheinen demgegenüber eher hemmend zu wirken. Dabei spielt der Leistungsstand eine untergeordnete Rolle, d.h. auch Schülerinnen und Schüler, die beide noch wenig zu einem Problem wissen, können gemeinsam die Aufgabe lösen. Aus der Perspektive Vygotskys und dessen Begriff der "Zone der proximalen Entwicklung» dagegen ist für die kognitive Entwicklung des Individuums die Zusammenarbeit mit einer kompetenteren Partnerin als optimal zu betrachten.

Da sich unsere Dialogpaare aufgrund von Sympathie zusammensetzten, muss die dadurch entstandene Variation der Leistungszusammensetzung gesondert betrachtet werden. Es ergaben sich in Bezug auf das Vorwissen für die beiden Schlüsselprobleme folgende Dialogpartnerkonstellationen: Homogen waren $40 \%$ der Dialogpaare (Schiff) bzw. 46\% (Song). Wenig heterogen (Unterschied von einem Niveau) waren 40\% (Schiff) bzw. 43\% (Song). 20\% (Schiff) bzw. 11\% (Song) waren stark heterogen (Unterschied zwei Niveaus und mehr) 
Wie die Abbildungen 2 und 3 zeigen, bestehen bei den homogenen, den leicht und den stark heterogenen Paaren signifikant unterschiedliche Entwicklungen.

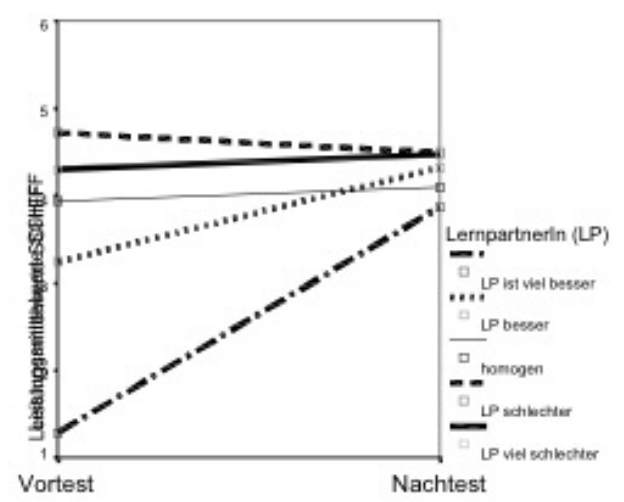

Abbildung 2: Schiff-Problem (N=310): Mittelwerte der Entwicklung von Vor-zu Nachtest in unterschiedlich zusammengesetzten Dialogpaaren. Die Dialogpartnerund-partnerinnen in den Dialogpaaren, die gleiches, heterogenes und stark heterogenes Vorwissen aufweisen, entwickeln sich unterschiedlich.

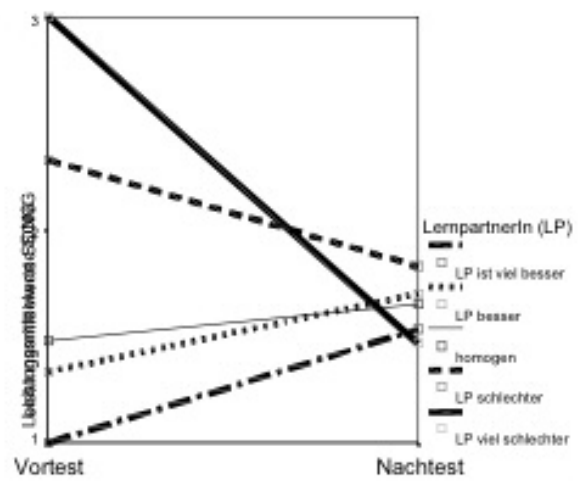

Abbildung 3: Song-Problem( $N=275)$ : Mittelwerte der Entwicklung von Vor-zu Nachtest in unterschiedlich zusammengesetzten Dialogpaaren. Die Dialogpartnerund-partnerinnen in den Dialogpaaren, die gleiches, heterogenes und stark heterogenes Vorwissen aufweisen, entwickeln sich unterschiedlich.

Die Varianzanalyse weist diese Entwicklungsunterschiede bei beiden Problemtypen als überzufällig aus, wobei sich sowohl ein Zeiteffekt als auch eine Interaktion zwischen der Entwicklung und der Lernpartnerzusammensetzung ergibt. 
Die meisten Lernenden, die beim Schiff-Problem mit einem wesentlich besseren Dialogpartner zusammengearbeitet hatten, veränderten ihre Einzelleistung im Nachtest positiv. Den deutlichsten Sprung nach oben machten beim naturwissenschaftlichen Problem die schwächeren Partnerinnen in den stark heterogenen Paaren. Die Unterschiede der Entwicklung dieser Gruppe zu allen anderen ist hoch signifikant. Die zweitstärksten Entwickler bildeten die schwächeren Partner in den schwach heterogenen Paaren - sie unterschieden sich aber nicht von den homogenen Paaren. Die vorwissensmässig relativ starken Schülerinnen zeigten Entwicklungen, die sich im Rahmen des Zufalls bewegten. Auffällig war der Ansatz von Regression, wenn die Dialogpartnerin leicht schwächeres Vorwissen hatte.

Dass die Dialogpaare, in denen die eine Partnerin ein höheres Vorwissen hatte, erfolgreicher arbeiteten, lässt sich als Beleg für die Wirkung der von Vygotsky (1978) beschriebenen «Zone der proximalen Entwicklung» betrachten - wobei bei ihm offen bleibt, wie «breit» diese Zone sein darf. Die Ergebnisse zum Schiff-Problem weisen darauf hin, dass in stark heterogenen Paaren vor allem der schwächere Schüler bzw. die schwächere Schülerin lernte.

Beim Song-Problem unterscheidet sich demgegenüber die Entwicklung der Lernenden aus homogenen Paaren nicht von den Schülerinnen und Schülern mit leicht oder viel besserem Lernpartner. Dafür ist die Regression der Schülerinnen und Schüler, die einen schlechteren Partner hatten, hoch signifikant.

Während also die Ergebnisse zum Song-Problem eine Bestätigung der Erwartungen aus der Entwicklungspsychologie in der Perspektive Piagets liefern, widersprechen die Ergebnisse zum Schiff-Problem diesem Ansatz. Dies ist umso verwunderlicher, als eher das physikalische als das sprachliche Problem starke entwicklungspsychologische Voraussetzungen hat.

Interessant sind die Detailunterschiede. Während beim Schiff-Problem in der Dialogsituation selbst noch kein Einfluss der Lernpartnerzusammensetzung auftritt, schneiden in der Lösung des Song-Problems die wenig heterogenen Dialogpaare signifikant besser ab als die homogenen und die stark heterogenen.

Eine umstrittene Frage betrifft den Nutzen kooperativen Lernens für die jeweils leistungsstärkeren Partner und Partnerinnen. In unserer Untersuchung liess sich bestätigen, dass auch der stärkere Partner profitieren kann, allerdings gilt dies nicht generell:

Von den 91 Schülerinnen und Schülern, die beim Schiff-Problem einen - nach Vortest - schwächeren Partner oder eine schwächere Partnerin hatten (das waren $31 \%$ aller beteiligten Schülerinnen und Schüler), entwickelten sich 18 weiter, also knapp 20\%. Ihnen stehen 21, d.h knapp 25\% gegenüber, die im Nachtest schlechtere Ergebnisse aufweisen, die übrigen veränderten sich nicht.

Wesentlich schlechter sieht die Bilanz beim Song-Problem aus: Von den 73 Schülerinnen und Schülern mit schwächeren Partnern oder Partnerinnen (26\% der Gesamtgruppe) zeigten 9, also 12\% einen Fortschritt, 63\% einen Rückschritt (die Hälfte davon regredierten allerdings nur wenig) und $25 \%$ veränderten sich nicht. 
Hier ergeben sich interessante Zusammenhänge zu Studien von Tudge (1992): Kindergarten- und Unterstufenkinder wurden von Tudge anhand der bekannten Balkenwaage aus Piagets Untersuchungen daraufhin untersucht, ob sie die Bewegung der Balkenwaage richtig voraussagen konnten. Die Antworten der Kinder wurden mit sieben hierarchisch aufeinander aufbauenden Regeln erfasst. Nach der niedrigsten Regel zeigen die Kinder überhaupt kein Verständnis für die richtigen Zusammenhänge zwischen der Anzahl der Gewichtssteine an der Balkenwaage und der Distanz dieser Gewichte nach links und nach rechts vom Angelpunkt der Waage aus gesehen (Regel 0). Mit der höchsten Regel verfügten sie über ein vollständiges Verständnis dafür (Regel 6). Die übrigen fünf Regel verteilen sich systematisch dazwischen.

Nach diesem Vortest arbeiteten die Kinder zur gleichen Aufgabe zu zweit zusammen. Ihre Aufgabe bestand wiederum darin, je einzeln vorauszusagen, wie sich die Balkenwaage verhalten würde, nachdem der Versuchleiter eine bestimmte Gewichts-Abstands-Konfiguration hergestellt hatte. Stimmten ihre Voraussagen nicht überein, mussten sie sich auf eine gemeinsame Aussage einigen. Die Kinder wurden so zu einem Paar zusammengesetzt, dass Interaktion mit einem Partner mit gleichem, mit tieferem oder mit höherem Regelniveau möglich war.

Die Ergebnisse fielen, je nach Partnerin, ganz unterschiedlich aus. Am auffälligsten war, dass in überraschend hohem Ausmass auch Regression vorkam: Etwa ein Drittel der Kinder, die mit einem gleich starken Partner, und beinahe die Hälfte der Kinder, die mit einem schwächeren Partner zusammengearbeitet hatten, fielen auf eine niedrigere Regel zurück, als jene, die sie im Vortest verwendet hatten. Fortschritte machten vor allem diejenigen Kinder, die mit einem starken Partner zusammenarbeiten konnten, vergleichbar mit unseren Ergebnissen zum Schiff-Problem.

Offensichtlich wirkt sich die Dialogpartner-Zusammensetzung je nach Problemtyp, und wahrscheinlich auch je nach Stand des Vorwissens in Bezug auf das zu lösende Problem unterschiedlich aus. Während sich im offenen, divergenten Song-Problem (das den Schülerinnen und Schülern im Vergleich zum Schiff-Problem wesentlich weniger vertraut sein konnte, weil Probleme dieser Art in der Schule kaum vorkommen) die entwicklungspsychologisch zu erwartenden Vorteile der kleinen Unterschiede für die leistungsstarken Dialogpaare bestätigten, wirkten sich im konvergenten Schiff-Problem die grossen Unterschiede in der Leistungsfähigkeit der Dialogpartner und -partnerinnen im Sinne der Bedeutung des soziokulturellen Kontextes nach Vygotsky günstig aus. Dies ergänzt die jüngst von Fuchs, Fuchs, Hamett und Kerns (1998) publizierten Ergebnisse, wonach leistungsstarke Schülerinnen und Schüler bei der Bearbeitung von komplexen Problemen dann am meisten profitieren, wenn ihre Dialogpartner oder -partnerinnen ebenfalls zur leistungsstarken Gruppe gehören (homogen, leistungsstark). 


\section{Geschlechtsspezifische Unterschiede}

Von den durch die Schülerinnen und Schüler gebildeten Dialogpaaren waren über 95\% mit zwei Jungen oder zwei Mädchen besetzt. Dies bot Gelegenheit für die empirische Erfassung geschlechtsspezifisch begründbarer Unterschiede in der Lernleistung von Dialogpaaren. Zum einen liessen die geschlechtshomogenen Dialogpaargruppen ähnliche Effekte erwarten, wie sie aus neueren Studien bekannt sind: Seeduzierter Unterricht bringt vor allem in den naturwissenschaftlichen Lernbereichen Vorteile für die Mädchen. Die Bearbeitung eines physikalischen und eines sprachlichen Problems rückte zudem die Unterrichtsdisziplinen in den Blickwinkel. Gemäss verschiedener Untersuchungen ist die Variable Geschlecht unterschiedlich wirksam, je nach Inhaltsbereich, aus dem das zu bearbeitende Problem stammt (Moser, Ramseier, Keller und Huber, 1997; Faisst, Häussler et al., 1994; Mantovani Vögeli, 1994).

Unsere Ergebnisse zeigen, dass die Mädchen sich beim Schiff- und beim SongProblem signifikant stärker entwickelten als die Jungen. Beim Schiff-Problem erreichten sie zwar nicht das Leistungsniveau der Jungen, aber sie verzeichneten den grösseren Entwicklungszuwachs (siehe Abbildung 4).

Beim Song-Problem starten die Mädchen leicht höher als die Jungen und entwickeln sich positiv, während die Jungen im Nachtest schlechter abschneiden. Beim Schiff-Problem können die Mädchen einen Rückstand beim Start deutlich verringern. Beide Effekte können als überzufällig bestätigt werden (Schiff: $F=4.3$, Sig.=.039, Song: F=5.3, Sig.=0.022).
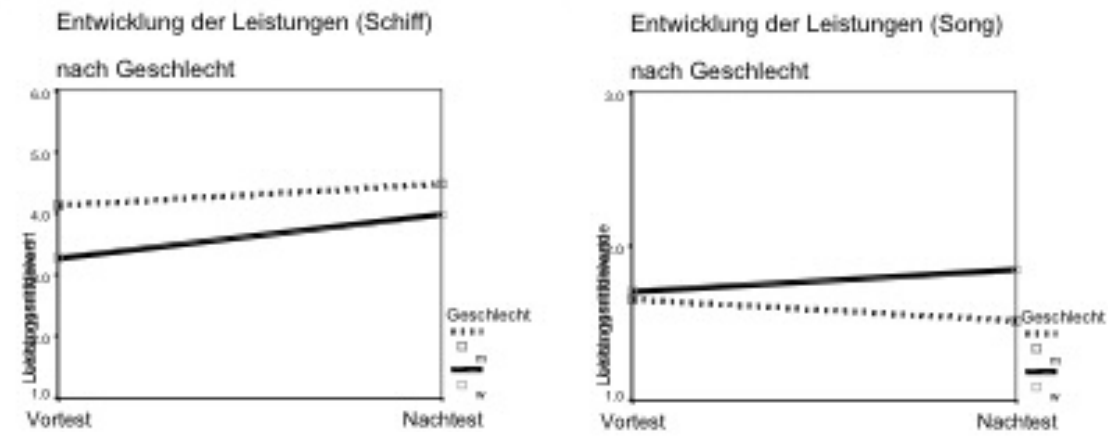

Abbildung 4: Entwicklung der Mittelwerte zwischen Vor-und Nachtest im Vergleich der Geschlechter

Dass für die Lernleistung das Geschlecht im insgesamt positiven Einfluss des Lernens im Dialog tatsächlich eine Rolle spielt, ist bemerkenswert.

Geschlechtsspezifischen Unterschieden, die vor allem in den naturwissenschaftlichen Lernbereichen im koedukativen Unterricht zu Ungunsten der Mädchen auftreten, wurde bisher durch einen teilweise seeduzierten Unterricht (BrockUtne und Hauka, 1986; Faulstich-Wieland, 1991; Faisst, Häussler et al., 1994), 
durch die Sensibilisierung der Lehrkräfte der naturwissenschaftlichen Fächer für das Problem sowie durch curriculare Massnahmen zu Gunsten von «mädchengerechteren» Inhalten zu begegnen versucht (Faisst, Häussler et al., 1994; Herzog, 1997, 1999).

Das in der vorliegenden Untersuchung praktizierte Verfahren der geschlechtshomogenen Dialogpaare stellt dazu eine interessante Alternative dar. In einem koedukativen Unterricht ist das geschlechtshomogene Dialogpaar kurzzeitig ein Element des seeduzierten Unterrichts, ohne dass dadurch der ganze Unterricht seeduziert durchgeführt werden müsste.

\section{Gewissheitsorientierung}

Studien von Huber, Sorrentino et al. (1992) machen deutlich, dass gewissheitsorientierte Schülerinnen und Schüler eher Mühe bekunden, sich in kooperativen Arbeitsformen zurecht zu finden. Ungewissheitsorientierte Lernende profitieren beim Lernen mit anderen dagegen mehr. Bei freier Wahl durch die Schülerinnen und Schüler finden sich in Arbeitsgruppen ungewissheitsorientierte und gewissheitsorientierte Lernende unter sich zusammen. In diesen Studien wurden Vorteile der ungewissheitsorientierten Schülerinnen und Schüler bei sprachlichen Aufgaben festgestellt.

In unserer Untersuchung fanden sich in etwa 30\% aller Dialogpaare Lernende mit gleicher Gewissheitsorientierung zusammen. Während diese Tendenz, sich mit Gleichgesinnten zusammen zu tun, nicht so stark bestätigt werden konnte, zeigte sich deutlich, dass ungewissheitsorientierte Jugendliche intelligenter sind als gewissheitsorientierte. Intelligenz und Art der Gewissheitsorientierung erwiesen sich als wichtigste Prädikatoren für die Lösung der beiden Schlüsselprobleme in der Dialogsituation.

Bestätigen wiederum liess sich die in anderen Studien festgestellten Vorteile ungewissheitsorientierter Schülerinnen und Schüler in Lernsituationen mit sprachlichen Aufgaben: Ungewissheitsorientierte Lernende schnitten beim SongProblem in der Dialogsituation signifikant besser ab als ihre gewissheitsorientierten Kolleginnen und Kollegen. Vermutlich interpretierten sie die Übersetzungsarbeit beim Song-Problem als offener als die Fragestellung zum physikalischen Problem, hinter dem sie - zu Recht - eine «richtige» Lösung vermuteten. Damit scheinen die Schülerinnen und Schüler, die mit unstrukturierten Anforderungen weniger Mühe bekunden, im Dialog im Vorteil gewesen zu sein.

\section{Diskussion und Folgerungen}

Unsere Ergebnisse bestätigen insgesamt die Vermutung, dass die Dialogsituation für das Lernen des einzelnen fruchtbar ist. Allerdings gibt es Ausnahmen, Fälle dialogischen Lernens, wo kein Fortschritt gemacht wurde oder sogar Regression vorkam. Der Dialog ist als Lernsituation psychologisch nicht einfach dadurch zu 
beschreiben, dass innere und äussere Wirkfaktoren bestimmt werden und deren Zusammenspiel verallgemeinert wird. Vielmehr sind diese Faktoren in Abhängigkeit von der Konstellation der Dialogpartner, vom Lernbereich, vom Problemtyp und vom Vorwissen in Bezug auf das Problem auf je unterschiedliche Weise wirksam. Die Komplexität des Lernens im Dialog kann auf der Grundlage unserer Ergebnisse nur teilweise reduziert werden, indem die Wirkfaktoren im Zusammenhang mit den sie bestimmenden Rahmenbedingungen, d.h. in Bezug auf die einzelnen Situationen, in denen die Dialoge untersucht wurden, beschrieben und interpretiert werden.

\section{Die Aufgabenstruktur der beiden Problemtypen}

\section{Die konvergente Aufgabenstruktur des Schiff-Problems}

Im konvergenten physikalischen Problem des Typs «Warum schwimmt ein Eisenschiff?» (Schiff-Problem) spielte das Vorwissen der Dialogpartner und -partnerinnen eine entscheidende Rolle. Erfolgreich verlaufene Dialoge zeichneten sich dadurch aus, dass durch die Lernenden signifikant mehr Sachreferenzen aktiviert wurden als in weniger erfolgreichen Dialogen. Das unterschiedliche Vorwissen der Lernenden wurde in der Dialogsituation zum Teil durch die ins Gespräch gebrachten Sachreferenzen ausgeglichen, was denselben Effekt hatte wie wenn ein mehr wissender Dialogteilnehmer seinen weniger wissenden Dialogpartner instruieren würde. Wenn auch diese Dialoge zwischen heterogenen Dialogpartnern nicht den Charakter eines Instruktionsdialoges hatten, zeigten sie doch denselben Effekt des teilweisen Ausgleichs im unterschiedlichen Vorwissen, d.h. beiläufiges Lernen (incidental learning) fand statt.

Die aus der einschlägigen Fachliteratur bekannten charakteristischen Merkmale eines produktiven Lerndialogs wie etwa steuernde Anmerkungen im Sinne der Metakognition oder erkenntnisfördernde Interaktionen (z.B. Transakte) und Argumentationen zur Problemlösung (z.B. Hypothesen, Bilanzierungen) erwiesen sich in den von uns in den öffentlichen Schulen aufgenommenen und analysierten Dialogen nicht als bedeutsame Wirkfaktoren. Das heisst aber nicht, dass sie es nicht sein könnten, wenn ihr Gebrauch in einer Interventionsstudie entsprechend trainiert würde. Offensichtlich wirkte in dem von uns untersuchten konvergenten physikalischen Problem die Sachstruktur als wirksame implizite Steuerung im Problemlösedialog, so dass keine explizite Steuerung erforderlich war oder der wissendere Partner bzw. Partnerin sie mit seinem Vorgehen nahe legte. Das von beiden Lernenden aufgrund ihres Vorwissens und im Dialog aktualisierte Sachwissen führte zu einem Erkenntnisfortschritt, ohne dass die Verknüpfung der verschiedenen Sachreferenzen durch die einzelnen Dialogpartner explizit geäussert werden musste. Daher traten die interaktiven Faktoren (z.B. Transakte in Form von Widerlegungen und Weiterführungen) auch nicht als bedeutsame Wirkfaktoren auf. 
Die Analyse der Wirkfaktoren in den Dialogen zu diesem Problem ergab darüber hinaus das überraschende Ergebnis, dass sachfremde Äusserungen (z.B. spassige Bemerkungen) einen bedeutsamen Indikator für erfolgreiches gemeinsames Problemlösen darstellen. Offensichtlich wiesen diese sachfremden Äusserungen darauf hin, dass der Dialog sich in gelöster Atmosphäre und guter Stimmung konstruktiv bewältigen liess. Wo dagegen aufgrund ungenügenden Vorwissens und zu grosser Unvertrautheit mit dem Problem und der Art seiner Bearbeitung Überforderung droht, stellen die sachfremden Äusserungen einen bedeutsamen Indikator für erlebens- statt aufgabenbezogener Informationsverarbeitung dar.

Ebenfalls als nicht bedeutsam erwiesen sich die Kategorien des Problemlösens. Hier vermuten wir, dass Dialoge unter Erwachsenen, die bewusster argumentieren können als Jugendliche im Alter unserer Probanden, andere Ergebnisse erwarten liessen. In unserer Studie konnten die analysierten Problemlösekategorien (Erklären, Hypothese, Bilanz) nicht als statistisch relevante Wirkfaktoren im Dialog nachgewiesen werden.

\section{Die divergente Aufgabestruktur des Song-Problems}

Gerade umgekehrt wie beim konvergenten Schiff-Problem präsentieren sich die Ergebnisse beim Songproblem: Es wurde eine hohe, allerdings nicht signifikante Korrelation zwischen expliziten Steuerungsäusserungen und dem Dialogergebnis ermittelt. Zwischen den aufgabenfremden Äusserungen und dem Dialogergebnis besteht eine signifikant negative Korrelation mit dem Dialogergebnis. Unseres Erachtens hat das ganz wesentlich damit zu tun, dass es sich beim Songproblem um ein divergentes Problem handelt.

Bei der Anwendung von Verfahren der Textüberarbeitung geht es in erster Linie nicht um das Finden und Sich-Einigen auf «richtige» Sätze bzw. Übersetzungen, sondern um die Passung einzelner Übersetzungssegmente in den umfassenderen Kontext. Dabei mussten sowohl formal-stilistische als auch inhaltliche Aspekte im Blick behalten und diskutiert werden. Die Passung eines Textsegmentes musste grundsätzlich also immer den «ganzen» Text im Blick haben. Es versteht sich, dass solche Problemlöseprozesse schon im monologischen Problemlösen anspruchsvoll sind. Bei der Arbeit im Dialog wird der Grad an Komplexität zusätzlich dadurch erhöht, dass gleichsam zwei individuelle Überarbeitungskonzepte von den Lernenden parallel berücksichtigt werden müssen.

Der Problemlöseprozess als solcher kann nur sehr bedingt durch die Sache implizit - nämlich das Textüberarbeiten - gesteuert werden. Es gibt als Referenzgrösse nicht «die» richtige Textüberarbeitung im Sinne eines Lehrsatzes. Den Lernenden stehen lediglich die Instrumente der Textüberarbeitung mehr oder weniger reichhaltig zur Verfügung. Diskutiert werden muss also immer wieder die Anwendung instrumentellen Wissens mit Blick auf die Qualität des Produktes, wobei gerade für die Beurteilung dieser Qualität keine fixe Referenzgrösse zur Verfügung steht. 
Explizite Steuerung wird von den Lernenden in der Konfrontation mit diesem divergenten Problem offensichtlich nicht als Unterbrechung des Problemlöseprozesses erlebt, sondern als von Fall zu Fall notwendige Orientierungs- oder Evaluationshandlung erfahren. Unterbrochen wird der Problemlöseprozess also dann, wenn er für die Lernenden zu komplex und unübersichtlich wird. Die Unterbrechung ist alles andere als "freiwillig», sondern schlichte Notwendigkeit. Aufgabenfremde Äusserungen unterbrechen den Problemlöseprozess, ohne diesen dadurch weniger komplex oder übersichtlicher werden zu lassen. Im Gegenteil: sie bergen die Gefahr, dass der Problemlöseprozess zwar nicht komplexer, aber mit Sicherheit unübersichtlicher wird. Die Tatsache, dass aufgabenfremde Äusserungen signifikant negativ mit dem Dialogergebnis korrelieren, unterstützen diesen Erklärungsversuch ebenso wie die Tatsache, dass explizite Steuerungsäusserungen hoch positiv, wenn auch nicht signifikant mit diesem korrelieren.

\section{Mädchen sind in geschlechtshomogenen Dialogpaaren stärker}

Werden die Dialogpaare durch Mädchen gebildet, fallen die Lernhemmnisse weg, die auf bestehende Geschlechterunterschiede zurückzuführen sind. Die Mädchen konnten sich in gleichgeschlechtlichen Dialogpaaren ohne Beeinträchtigung aufgrund der Anwesenheit von Jungen mit den Problemen auseinandersetzen. In beiden Problemen zeigten die Mädchen denn auch einen grösseren Entwicklungsfortschritt als die Jungen, und zwar trotz der für die Mädchen im Bereich des naturwissenschaftlichen Problems deutlich ungünstigeren Lehrererwartungen. Die Dialogsituation in gleichgeschlechtlichen Dialogpaaren brachte den Mädchen eindeutig grössere Lernchancen.

Beachtet man die Vorteile, die Mädchen in gleichgeschlechtlichen Dyaden in unserer Untersuchung hatten, dürften Lehrpersonen nicht mehr auf die Idee kommen, die Dialogpaare aus disziplinarischen Gründen gemischtgeschlechtlich zu bilden. Sie müssten im Gegenteil dafür sorgen, dass Mädchen und Jungen vermehrt in Dialogsituationen gleichgeschlechtliche Partnerschaften bilden dürfen. Beachtet man den Einfluss, den Erwartungen auf die Leistungsfähigkeit der Schülerinnen und Schüler haben, müssten die Lehrpersonen gerade ihre geschlechtsspezifischen Leistungserwartungen dringend überprüfen.

\section{Zusammensetzung der Dialogpaare - Bedeutung der Vorwissensbestände}

Deutliche Unterschiede in den Leistungen im Dialog und im Nachtest waren auf die unterschiedlichen Voraussetzungen der Lernenden zurückzuführen. In heterogenen Dialogpaaren profitierten vor allem die schwächeren von den stärkeren Dialogpartnern; ebenso machten homogene Dialogpaare, bei denen beide Partner nach ihrem Vorwissen eher schwächer eingeschätzt waren, Fortschritte. Dies darf nicht zum falschen Schluss führen, stärkere Dialogpartnerinnen würden nicht profitieren. 20\% dieser Schülerinnen beim Schiff-Problem und 12\% beim 
Song-Problem machen Fortschritte, obwohl sie als die besseren Dialogpartner schon im Vortest hohe Leistungen erbrachten, womit die Wahrscheinlichkeit zur Weiterentwicklung geringer wurde.

Intelligenz und Ungewissheitsorientierung als wirksame personale Bedingungen

Für beide Probleme erwiesen sich Intelligenz und Ungewissheitsorientierung als wichtige Prädiktoren für die Problemlösung. Ungewissheitsorientierung war zudem ein bedeutsamer Vorteil für die Bearbeitung des sprachlichen Problems. Diese Ergebnisse entsprechen anderen Studien, die insbesondere für ungewohnte, divergent angelegte Probleme einen positiven Einfluss für Schülerinnen und Schüler entdeckt haben, die solche Aufgaben als Herausforderung betrachten und mit der Unsicherheit der Situation in der Lage sind umzugehen.

\section{B i l anz}

Die Bilanz muss nach Dialogpartnerzusammensetzungen unterschieden werden:

\section{Lernen mit einem kompetenteren Partner an einem konvergenten Problem}

Wenn unterschiedlich kompetente Dialogpartner zusammen arbeiten, sind sie erfolgreich,

- wenn der kompetentere Partner von seinem besseren Wissen im Dialog tatsächlich Gebrauch macht, d.h. wenn sich dieser seines qualitativ besseren Wissens so sicher ist, dass er im Dialog vom schwächeren Partner nicht verunsichert wird,

- wenn der Dialog in guter Stimmung stattfindet,

- wenn die Dialogpartner eine Vielzahl von unterschiedlichen Sachreferenzen gedanklich durcharbeiten und

- wenn sie mit gleichgeschlechtlichen Dialogpartnern zusammenarbeiten,

- und vermutlich auch, wenn die Dialogpartner die Möglichkeit haben, in handelnder Erprobung bestätigende oder falsifizierende Rückmeldungen auf ihre Lösungsversuche zu gewinnen (was zwar nicht systematisch überprüft wurde, sich aber in den Videos der Dialogpaare zum Schiff-Problem beobachten liess und sich auch mit Erfahrungen von Tudge (1992) deckt).

Lernen in einem Leistungsstarken Dialogpaar an einem divergenten Problem

Wenn zwei leistungsstarke Partner oder Partnerinnen, die sich mit einem divergenten Problem auseinandersetzen, eine Dyade bilden, sind sie erfolgreich,

- wenn beide Dialogpartner in Bezug auf das zu lösende Problem gemeinsam über vielfältiges und reichhaltiges Vorwissen (Breite und Differenzierungsgrad des Vorwissens) verfügen; 
- wenn sich dieses elaborierte Wissen aus dem bei den Dialogpartnern individuell bestehenden elaborierten Wissensbeständen zusammensetzt und damit die für die Problemlösung zur Verfügung stehende Wissensbasis (Konsolidierung des Vorwissens) vergrössert und verstärkt;

- wenn das Dialogpaar eher ungewissheits- als gewissheitsorientiert ist;

- wenn die Dialogpartner eher überdurchschnittlich intelligent sind.

\section{Ausblick}

Ein Problem in einer Dialogpartnerschaft zu lösen, muss noch nicht in jedem Fall bedeuten, dass Lernfortschritt erzielt wird. Was in der produktiven Phase eines dialogischen Problemlösens erfolgt ist, bedarf der Reflexion, des Durcharbeitens und der wiederholenden und wiederholten Anwendung, damit wirklich gelernt wird. Es mag dazu durchaus notwendig sein, dass kompetentere Lernende und die Lehrperson zur Lösung eines Problems bzw. zur Lösung von Aufgaben, ja überhaupt zur Begleitung und Förderung von Lernprozessen (im Sinne von "guidance»), am dialogisch ablaufenden Lernen Anteil nehmen (Wagenschein, 1982; Palincsar und Brown, 1984; Baer, 1998; Brown und Campione, 1998; Stebler, 1999; Schneeberger, in Vorb.).

\section{Metakognition auslösen}

Die in unserer Untersuchung gemachte Erfahrung, dass Metakognition, Interaktionsstrukturen und Problemlöseargumentationen wenig Einfluss auf die dialogischen Problemlöseprozesse hatten, darf nicht dazu verleiten, diese Strukturierungs- und Reflexionshilfen zu vernachlässigen. Vielmehr müssten die Lehrpersonen dafür sorgen, dass die Dialogfähigkeit der Schülerinnen und Schüler durch entsprechende Förderung und Reflexionen erweitert und ausgebaut werden kann. Wir untersuchten Dialoge vor allem in der Produktionsphase. Das heisst aber nicht, dass nach einem Dialog durch eine entsprechende Reflexion und durch geeignete Formen des Erfahrungsaustausches, der Evaluation und des Conferencings nicht bewusst die Dialogfähigkeit gesteigert werden könnte (Wegerif, Mercer und Dawes, 1999).

\section{Lernen im Dialog braucht Akzeptanz bei den Lehrenden}

Sowohl eine vorbereitende repräsentative Umfrage unter Oberstufenlehrkräften im Kanton St. Gallen als auch die Befragung der Lehrpersonen, deren Klassen an unserer Untersuchung teilnahmen, zeigen, dass die Möglichkeiten des Dialogs noch zu wenig genutzt werden und dass bei den Lehrenden oft Skepsis gegenüber dialogischen Formen des Lernens besteht. In Anbetracht der potenziellen Lernmöglichkeiten, welche Dialogsituationen bieten, müsste eine grössere Akzeptanz 
bei der Lehrerschaft erwartet werden können. Dabei ist auch zu beachten, unter welchen Bedingungen Lernen im Dialog am erfolgreichsten ist.

Auf diesem Hintergrund liegt die Vermutung nahe, dass dialoggewohntere Schülerinnen und Schüler erfolgreicher gearbeitet hätten als diejenigen in unserer repräsentativen Stichprobe. Dies wird vor allem durch die festgestellten Klassenunterschiede (die hier nicht dargestellt wurden) gestützt. Die Schulkultur müsste also dialogfreundlicher gestaltet werden; Lehrkräften müssten dialogische Aktivitäten der Schülerinnen und Schüler besonders am Herzen liegen.

\section{Anmerkungen}

1 Ausgelassene Dialogteile, Pausen und andere Transkriptnotizen sind der Lesbarkeit wegen in diesem Auszug weggelassen worden.

2 Bei den Einschätzungen eines Auswerterpaares wurden eine annehmbare Übereinstimmung von rund $80 \%$ erzielt. Abweichende Einschätzungen wurden von den jeweiligen zwei Auswerterinnen bzw. Auswertern in einem gemeinsamen Einigungsprozess bereinigt.

3 Beispiele für diese Kategorie: "Wasser ist stark» / "Ein Eisenschiff schwimmt doch garnicht!»

\section{Literatur}

Abele, A. (1995). Stimmung und Leistung. Göttingen: Hogrefe.

Anderson, J.R., Reder, L.M. \& Simon, H.A. (1996). Situated learning and education. Educational Researcher, 25(4), 5-11.

Baer, M. (1998). Textverfassen als beobachtbarer und als förderungsfähiger Prozess. Habilitationsschrift, Universität Zürich: Philosophisch-historischen Fakultät.

Beck, E., Bachmann, T., Geering, P., Guldimann, T., Niedermann, R., Uhland Mogg, E., Wigger, A., Zutavern, M. (1992). Projekt Eigenständige Lerner. Wissenschaftlicher Schlussbericht an den Schweizerischen Nationalfonds. (Bericht 8) . Pädagogische Hochschule St. Gallen: Forschungsstelle.

Beck, E., Guldimann, T. \& Zutavern, M. (Hrsg.) (1995). Eigenständig Lernen. St. Gallen: UVK.

Beck, E., Guldimann, T. \& Zutavern, M. (1997). Lernen im Dialog. (Bericht 15). In E. Beck, T. Guldimann \& M. Zutavern (Hrsg.), Lernkultur im Wandel. Tagungsband der Schweizerischen Gesellschaft für Lehrerinnen- und Lehrerbildung und der Schweizerischen Gesellschaft für Bildungsforschung (Schriftenreihe Kollegium, Bd. 4) (S. 163-177). St. Gallen: UVK.

Beck, E., Baer, M., Bachmann, T., Guldimann, T., Niedermann, R., Zutavern, M. (1998). Projekt "Lernen im Dialog». Schlussbericht an den Schweizerischen Nationalfonds zur Förderung der wissenschaftlichen Forschung. Pädagogische Hochsschule St. Gallen: Forschungsstelle.

Bell, N., Grossen, M. \& Perret-Clermont, A.-N. (1985). Sociocognitive conflict and intellectual growth. In M.W. Berkowitz (Hrsg.), Peer conflict and psychological growth (S. 41-54). San Francisco: Josey-Bass.

Berkowitz, M.W. (1982). The role of discussion in moral education. Paper presented at the International Symposium on Moral Education: Fribourg.

Brock-Utne, B. \& Hauka, R. (1986). Wissen ohne Macht. Frauen als Lehrerinnen und Schülerinnen. Giessen. 
Brown, A.L. \& Palincsar, A.S. (1989). Guided, cooperative learning and individual knowledge acquisition. In L.B. Resnick (Hrsg.), Knowing, learning, and instruction: Essays in Honor of Robert Glaser (S. 393-451). Hillsdale: Erlbaum.

Brown, A.L. \& Campione, J.C. (1998). Designing a community of young learners: theoretical and practical lessons. In N.M. Lambert \& B.L. McCombs (Hrsg.), How students learn: reforming schools through learner-centered education. Washington, D.C.: American Psychological Association.

Csikszentmihaly, M. (1991). Das Flow-Erlebnis. Jenseits von Angst und Langeweile im Tun aufgehen. Stuttgart: Klett-Cotta.

Csikszentmihaly, M. \& Csikszentmihaly, I.S. (Hrsg.) (1991). Die aussergewöhnliche Erfahrung im Alltag. Die Psychologie des Flow-Erlebnisses. Stuttgart: Klett-Cotta.

Dawes, L., Fisher, E. \& Mercer, N. (1993). The quality of talk at the computer. Language \& Learning, 22-25.

Dewey, J. (1916/1993). Demokratie und Erziehung. Eine Einleitung in die philosophische Pädagogik. Weinheim: Beltz.

Doise, W. \& Hanselmann, C. (1990). Interaction sociale et acquisition de la conservation du volume. European Journal of Psychology of Education, 5(1), 21-31.

Doise, W., Mugny, G. \& Perret-Clermont, A.N. (1975). Social interaction and the development of cognitive operations. European Journal of Social Psychology, 5(3), 367-383.

Doise, W. \& Mugny, G. (1984). The social development of the intellect. Oxford: Pergamon Press.

Ehlich, K. (1981). Schulischer Diskurs als Dialog? In P. Schröder \& H. Steger. (Hrsg.), Dialogforschung: Jahrbuch des Instituts für deutsche Sprache (Sprache der Gegenwart, Bd. 54) (S. 334-369). Düsseldorf: Schwann.

Faisst, W., Häussler, P. et al. (1994). Physik-Anfangsunterricht für Mädchen und Jungen . Universität Kiel: Institut für die Pädagogik der Naturwissenschaften.

Faulstich-Wieland, H. (1991). Koedukation - Enttäuschte Hoffnungen? Darmstadt: Wissenschaftliche Buchgesellschaft.

Foppa, K. (1994). «Verstehen im Dialog» und «Textverstehen»: Zwei Seiten einer Medaille? Überlegungen zu einem vernachlässigten Problem. In R. Reusser \& M. Reusser-Weyeneth (Hrsg.), Verstehen. Psychologischer Prozess und didaktische Aufgabe (S. 55-68). Bern: Hans Huber.

Fuchs, L.S., Fuchs, D., Hamlett, C.L., et al. (1998). High-achieving students' interactions and performance on complex mathematical tasks as a function of homogeneous and heterogeneous pairings. American Educational Research Journal, 35(2), 227-267.

Gilly, M., Fraisse, J. \& Roux, J.-P. (1988). Résolution de problèmes en dyades et progrès cognitifs chez des enfants de 11 à 13 ans: dynamiques interactives et mécanismes socio-cognitifs. In A.N. Perret-Clermont \& M. Nicolet (Hrsg.), Interagir et connaître: Enjeux et régulations sociales dans le dévelopement cognitif (S. 73-92). Fribourg: Del Val.

Gilly, M. \& Deblieux, M. (1995). Travail en dyade et selection des informations les plus importantes d'un texte narratif. Bern.

Glachan, M. \& Light, P. (1982). Peer interaction and learning: can two wrongs make a right? In G. Butterworth \& P. Light (Hrsg.), Social Cognition: Studies of the Development of Understanding (S. 238-262). Brighton: Harvester Press.

Glaser, R. (1991). The maturing of the relationship between the science of learning and cognition and educational practice. Learning and Instruction, 1(2), 129-144.

Golder, C. (1992). Production of Elaborated Argumentative Discourse: The Role of Cooperativeness. European Journal of Psychology of Education, 7(1), 51-59.

Helm, J. (1954). Über den Einfluss affektiver Spannung auf das Denkhandeln. Zeitschrift für Psychologie, 157, 23-105.

Helm, J. (1958). Über die Wirkung von Erfolgsserien auf das Denkhandeln und die Leistung. Zeitschrift für Psychologie, 162, 3-114. 
Herzog, W. (1999). Professionalisierung im Dilemma. Braucht die Lehrerinnen- und Lehrerbildung eine eigene Wissenschaft? Beiträge zur Lehrerbildung, 17(3), 340-374.

Herzog, W. et al. (1997). Koedukation im Physikunterricht. Schlussbericht an den Schweizerischen Nationalfonds zur Förderung der wissenschaftlichen Forschung. Universität Bern: Abteilung Pädagogische Psychologie und Abteilung für das Höhere Lehramt.

Huber, G.L., Sorrentino, R.M., Davidson, M.A., et al. (1992). Uncertainty orientation and cooperative learning: Individual differences within and across cultures. Learning and Individual Differences, 4(1), 1-24.

Krappmann, L. \& Oswald, H. (1985). Schulisches Lernen in Interaktionen mit Gleichaltrigen. Zeitschrift für Pädagogik, 31(3), 321-337.

Lambert, N.M. \& McCombs, B.L. (Hrsg.) (1998). How students learn: reforming schools through learner-centered education. Washington, D.C.: American Psychological Association.

Mantovani Vögeli, L. (1994). Fremdbestimmt zur Eigenständigkeit: Mädchenbildung gestern und heute. Chur: Rüegger.

Mercer, N. (1995). The guided construction of knowledge. Talk amongst teachers and learners. London: Multilingual Matters.

Metzger, W. (1957). Stimmung und Leistung. Münster: Aschendorff.

Moser, U., Ramseier, E., Keller, C., et al. (1997). Schule auf dem Prüfstand. Eine Evaluation der Sekundarstufe I auf der Grundlage der "Third International Mathematics and Science Study". Chur: Rüegger.

Palincsar, A.S. \& Brown, A.L. (1984). Reciprocal teaching of comprehension fostering and comprehension monitoring activities. Cognition and Instruction, 1(2), 117-175.

Palincsar, A.S., Stevens, D.D. \& Gavelek, J.R. (1989). Collaborating with teachers in the interest of student collaboration. Educational Research, 13(1), 41-53.

Pauli, C. (Hrsg.) (1994). Mathematische Textaufgaben lösen mit HERON: Eine Analyse von Schülerdialogen in einer kooperativen Lernsituation mit und ohne Unterstützung durch ein tutorielles System. Burgdorf: Eigenverlag.

Pauli, C. (1998). Computerunterstützte Schülerzusammenarbeit im Mathematikunterricht. Dissertation, Universität Zürich: Pädagogisches Institut.

Perret-Clermont, A.-N., Mugny, G. \& Doise, W. (1976). Une approche psychosociologique du dévelopement cognitif. Archives de Psychologie, XLIV, 171, 135-144.

Piaget, J. (1936/1969). Das Erwachen der Intelligenz beim Kinde. Stuttgart: Klett.

Rumpf, H. (1976). Unterricht und Identität: Perspektiven für ein humanes Lernen. München: Juventa.

Schneeberger, M. (in Vorb.). Kollaboratives Problemlösen im Mathematikunterricht. Dissertation, Universität Zürich: Pädagogisches Institut.

Slavin, R.E. (1990). Cooperative learning: theory, research, and practice. Englewood Cliffs: Prentice-Hall.

Stebler, R. (1999). Eigenständiges Problemlösen. Zum Umgang mit Schwierigkeiten beim individuellen und paarweisen Lösen mathematischer Problemgeschichten - Theoretische Analyse und empirische Erkundigungen (Erziehungswissenschaft, Bd. 28). Bern; Berlin: Schweizerischen Gesellschaft für Bildungsforschung.

Tudge, J. (1992). Vygotsky, the zone of proximal development, and peer collaboration: Implications for classroom practice. In L.C. Moll (Hrsg.), Vygotsky and education: Instructional implications and applications of sociohistorical psychology (S. 155-172). Cambridge, New York: Cambridge University Press.

Vygotsky, L.S. (1978). Mind in society. The development of higher psychological processes. Cambridge, MA: Harvard University Press.

Wagenschein, M. (1982). Verstehen Lehren. Genetisch - sokratisch - exemplarisch (7., durchges. Aufl.). Weinheim; Basel: Beltz. 
Wegerif, R., Mercer, N. \& Dawes, L. (1999). From social interaction to individual reasoning: an empirical investigation of a possible socio-cultural model of cognitive development. Learning and Instruction, 9 (6), 493-516.

Wunderlich, D. (1969). Unterrichten als Dialog. Sprache im technischen Zeitalter, 32, 263287.

\title{
Apprendre par le dialogue - Déscription et analyse de dialogues à l'intérieur de dyades d'élèves autour de la résolution de problème
}

\begin{abstract}
Résumé
L'article est consacré à une étude portant sur les dialogues entre deux élèves autour de la résolution d'un problème sans la conduite ou le soutien d'un enseignant. En abordant un problème tiré d'une situation quotidienne («Pourquoi un bateau en fer flotte-t-il ?»), il s'agit de rejoindre un problème de physique. Le dialogue à l'intérieur de la dyade déclenche l'apparition de problèmes de fonds selon les expériences, des idées de solution et des questions réciproques. C'est ce qui constitue la productivité de telles situations.

L'étude représentative portant sur plus de 400 élèves de 20 classes de 8ème année scolaire analyse les dialogues à l'intérieur de deux situations: l'une portant sur un problème physique et l'autre sur un problème purement linguistique. L'objectif est de repérer les conditions de base qui contribuent à un dialogue plus ou moins profitable. Sont pris en compte notamment le sexe des élèves et le niveau de connaissance préalable des sujets. Un accent particulier a été mis sur les interactions langagières entre enfants dans un échantillon restreint de 31 dyades. Les résultats ont montré, entre autre, que la capacité à se guider joue un rôle plus important dans le problème de nature linguistique. Dans le problème de sciences physiques, la diversité des connaissances préalables et la possibilité de «s'écarter du sujet de manière créative» semblent représenter des aides. Ces résultats permettent de tirer des conséquences concernant l'apprentissage coopératif et l'importance de l'apprentissage entre partenaires. Ils offrent également l'intérêt de mettre en évidence l'importance du dialogue dans les situations d'enseignement à l'école.
\end{abstract}

\section{Apprendimento attraverso il dialogo. Descrizione e analisi di dialoghi di coppie di allievi in procinto di risolvere problemi}

Riassunto

Lo studio qui presentato analizza dialoghi di allievi che, senza l'aiuto dell'insegnante, cercano di risolvere un problema. L'obiettivo di rispondere ad una questione del tipo "perché galleggia una nave costruita in ferro?» permette di mettere 
a fuoco la ricerca della soluzione del problema di fisica sottostante. Nel dialogo appaiono esperienze, idee di soluzione e interrogativi che rendono produttiva la situazione. In uno studio rappresentativo condotto su più di 400 allievi di 20 classi dell'ottavo anno, sono stati analizzati dialoghi prodottisi nell'affrontare una questione di fisica e una questione linguistica. Si è trattato in particolare di mettere a fuoco le condizioni che contribuiscono a rendere i dialoghi produttivi per l'apprendimento. Ad es. si è potuto dimostrare l'incidenza dell'appartenenza sessuale degli allievi e delle loro preconoscenze.

Con un gruppo più ridotto di 31 coppie sono state analizzate le locuzioni verbali. I risultati mostrano tra l'altro che nella soluzione di un problema linguistico sono utili le capacità di pilotare, mentre quando gli allievi affrontano problemi legati alle scienze naturali, entrano in gioco piuttosto le preconoscenze e le intuizioni creative. Da questi dati gli autori derivano indicazioni circa l'importanza dell'apprendimento cooperativo e tra partner. Più in generale discutono il ruolo del dialogo per l'insegnamento.

\section{Learning in dialogue - Description and analysis of student dialogues during the solving of a problem in a learning dyad.}

\section{Summary}

The following study examined dialogues in which two students tried to solve a problem together without the help of a steering and correcting teacher. The goal, to answer an everyday question such as, "Why does an iron boat float?» leads the way to the solution of a physical problem. The study-partners experiences, solution ideas, and questions in the discussion about the proposed subject, make up the fruitfulness of such a situation. In a representative study with over 400 male and female students of 20 eight-grade classes, such dialogues to a physical and rather linguistical problem area where examined. The aim here was to analyze the paradigms that contribute to a more or less enhancement of learning throughout the dialogues. As an example, it was possible to prove the genderspecific differences and the significance of pre-existing knowledge.

A smaller spot-test of 31 dialogue-couples scrutinizes their conversations. The results show, among other things, that for the solution of a linguistical problem, the capability to steer matters, where with the natural-science task, the diversity of pre-existing knowledge and "creative straying» is helpful. From that, we can deduce conclusions for co-operative learning and the didactic meaning of studypartnerships. The significance of dialogues can as well be thematically examined in present school lessons. 